\title{
Design of probe for NQR/NMR detection
}

\author{
Preeti Hemnani $^{1}$, A. K. Rajarajan ${ }^{2}$, Gopal Joshi ${ }^{3}$, S. V. G. Ravindranath ${ }^{4}$ \\ ${ }^{1}$ Homi Bhabha National Institute, India \\ 2,3,4 Bhabha Atomic Research Centre, India \\ ${ }^{1}$ Department of Electronics and Telecommunication Engineering, S.I.E.S Graduate School of Technology, India
}

\begin{tabular}{l} 
Article Info \\
\hline Article history: \\
Received May 10, 2019 \\
Revised Dec 16, 2019 \\
Accepted Jan 11, 2020 \\
\hline Keywords: \\
Coil \\
FPGA \\
NMR \\
NQR \\
Probe
\end{tabular}

\begin{abstract}
Nuclear Magnetic Resonance (NMR) is a RF technique that is able to detect any compound by sensing the excited resonance signals from atomic nuclei having non-zero spin. NQR is similar to NMR but the only difference is NMR needs a DC magnetic field and due to this its application in the field is limited. A FPGA based NQR spectrometer is designed using a single FPGA chip to perform the digital tasks required for NQR spectrometer. Design of Probe for NMR/NQR spectrometer is researched. Parallel tuned and series tuned Probes are discussed and simulated.14N NQR from NaNO2 is observed from spectrometer designed with parallel tuned probe.
\end{abstract}

Copyright (C) 2020Institute of Advanced Engineering and Science. All rights reserved.

\section{Corresponding Author:}

Preeti Hemnani,

Department of Electronics and Telecommunication Engineering,

S.I.E.S Graduate School of Technology,

Nerul, Navi Mumbai, India.

Email: preetiahemnani@gmail.com

\section{INTRODUCTION}

Nuclear Quadrupole Resonance (NQR) is spectroscopy of nuclear energy levels resulting from the interaction of nuclear quadrupole moment with the electric field gradient in crystalline (non-cubic) solids. The nuclear energy levels pertaining to this interaction absorb and emit electromagnetic radiation in the RF range. In the pulsed NQR spectrometer, the sample is exposed to intense RF radiation of resonance frequency from which the nuclei absorb power. When the RF radiation is switched off the absorbed energy is radiated from the nuclei in a typical time constant $\left(\mathrm{T}_{2}{ }^{*}\right)$ of few tenths of microseconds to a few milliseconds. The resonance frequency depends on both the quadrupole moment and the electric field gradient hence is a property of not only the nucleus but also of the material. For most of the organic crystals the resonance frequency lies below $10 \mathrm{MHz} .{ }^{14} \mathrm{~N} \mathrm{NQR}$ frequencies for organic compounds like urea, NaNO2, RDX etc lies in the range of $1-5 \mathrm{MHz}[1-3]$. The explosive detection via NQR is also based on presence of ${ }^{14} \mathrm{~N}$ as all explosives usually contain nitrogen [4].

NQR is related to Nuclear magnetic Resonance (NMR) [5], which is also known by one of its applications, Magnetic Resonance Imaging (MRI). NMR is a spectroscopic technique which is used to detect the compounds by observing the resonance signal from atomic nuclei with non-zero spin inside the compound. As for NMR large magnetic field is required, its field application becomes limited and hence the NMR-based explosive detection systems are developed for luggage screening [6]. However, unlike NMR, the job of producing the spectroscopic energy levels in NQR is carried out by the crystalline electric field gradient and hence, $\mathrm{NQR}$ does not require a static magnetic field.

Originally conceptualized and observed by I. I. Rabi [7], technology of NMR developed in instrumentation and theory along with the development of RF technology during the world war and later commercial instruments for observation of NMR and Magnetic Resonance Imaging (MRI) were available for various companies. NQR however remained as a specialized field and the development in this field was 
limited to specialized applications like mine and drug detection. The theoretical development in NMR is very advanced to the extent that NMR devices are being developed for quantum computing purposes [8].

The NQR working principles are similar to those of NMR. NQR differs with NMR only in the way nuclear magnetic moment and the external magnetic field interacts, where unlike NMR, NQR exploits the interaction between the electric quadrupole moment of nucleus and EFG that surrounds the nucleus. Therefore, NQR does not require an external magnetic field. Atomic nuclei with spin I $>1 / 2$, such as $14 \mathrm{~N}$ $(\mathrm{I}=1), 17 \mathrm{O}(\mathrm{I}=5 / 2), 35 \mathrm{Cl}(\mathrm{I}=3 / 2), 63 \mathrm{Cu}(\mathrm{I}=3 / 2)$ possess a non spherical charge distribution (shape) and hence a quadrupole moment. In NQR, the electric quadrupole moment (denoted by Q) interacts with EFG of the non-cubic crystal site which, much like the Zeeman effect of NMR, results in the energy of the nucleus, depend on its orientation with respect to EFG. FPGA based pulsed NQR system is designed using parallel tuned probe [9]. Two type of probe circuits are available. Research was done on which type of probe to be used. This paper describes about the two types of probes and which to be used based on the study.

\section{FPGA BASED PULSED NQR SYSTEM DESIGN}

An NQR/NMR system[10,11], in general, is composed of four sections, transmitter, probe, receiver and computer control as shown in Figure 1. The transmitter section is designed to excite the nuclei using a high power RF pulse; hence it is comprises of an RF source, a pulse programmer and a power amplifier. The receiver section is designed to receive weak signal following the strong excitation and it comprises of a preamplifier, detectors, filters. The computer control could produce the required pulse sequence for excitation of nuclei; however it is designed to acquire, digitize, store and process the data. In highly sophisticated commercial systems the computer control module runs the entire process.

FPGA based pulsed ${ }^{14} \mathrm{~N}$ NQR spectrometer which is developed as a part of the work is explained in [12-14]. FPGA chip contains large number of programmable logic gates in which any of the digital circuits can be designed using hardware description languages like VHDL and Verilog. The digital tasks of this spectrometer like pulse programmer (PP), Direct Digital Synthesizer (DDS) [15, 16], digital demodulator [17], low pass Finite Impulse Response filter (FIR) [18] are designed using single chip of FPGA. The analog peripherals such as cross diodes, pre-ampifier, quarter wave transformer and probe were also designed as part of this research. This paper explains the design and development of probe for the NMR/NQR spectrometer.

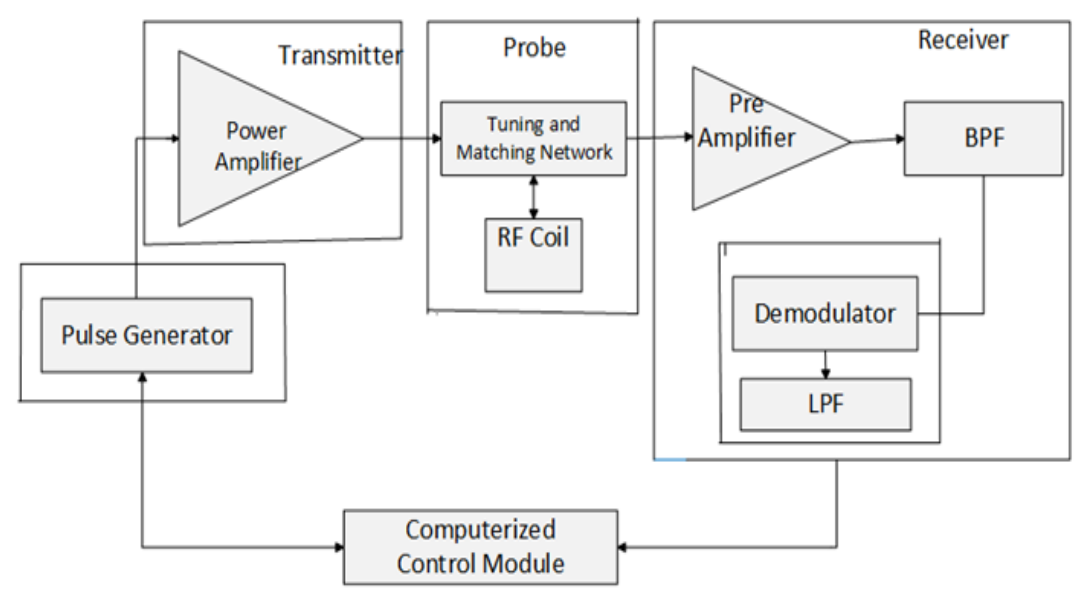

Figure 1. Block diagram of pulsed NQR pectrometer

\section{NMR/NQR PROBE}

The job of an NQR probe is twofold. It is required to produce the RF magnetic field that is sufficiently strong to excite the nuclei and required to be sensitive enough to detect the weak signal after the excitation is complete. The most suitable probe circuit for this purpose is the tuned LCR tank circuit. The Figure 2 shows series and parallel tank circuits that can be used for this purpose. Since in the tuned condition, the tank circuits have extreme impedances (zero or infinity theoretically), the transfer of power into the coil as well as transfer of signal from the coil require additional components that could match the impedance of these circuit to the output/input impedances of the amplifiers. This is typically achieved by 
making use of standard impedance configuration for the components in the circuit and matching the probe impedance to that value. The author has used $50 \mathrm{Ohm}$ components where as $75 \mathrm{Ohm}$ standard configurations are also available commercially.
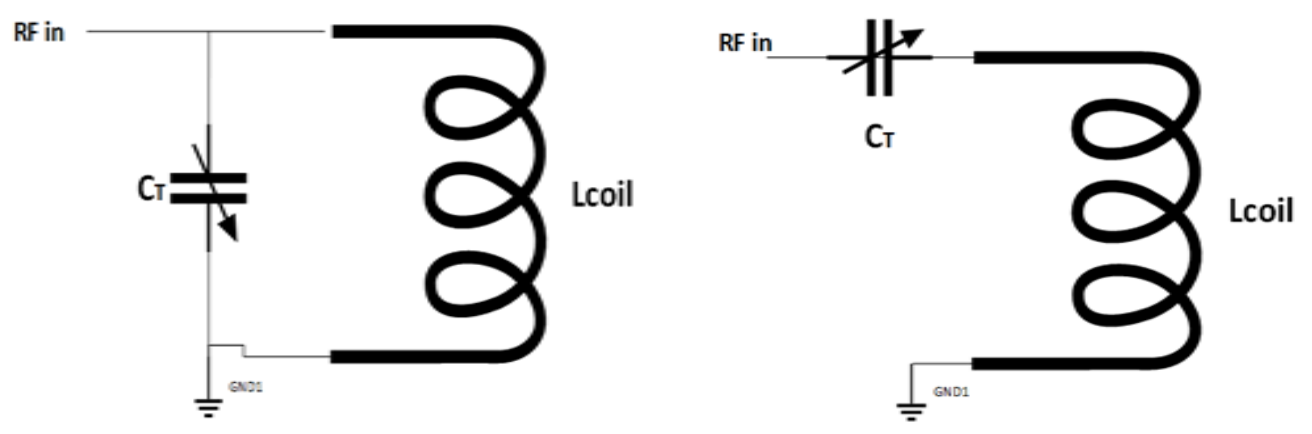

Figure 2. The parallel and series LC probe

The Q factor of the tank circuit plays an important role in amplifying the exciting signal further. However this is both a boon and a bane as the voltages in the coil could easily go beyond the breakdown voltages of the various components of the circuit and result in breakdown or sparking etc. So the probe needs to have a good insulation and need to use components with high voltage ratings wherever required. A coil always has a resistance associated with it and the same serves as a damping to the $\mathrm{Q}$ of the circuit. One may add additional resistances to the circuit in order to further dampen the $\mathrm{Q}$ or for the purposes of matching the impedance of the probe with rest of the electronics. The high $\mathrm{Q}$ is also associated with a long recover time (ringing) of the probe. Additional circuitry can be used in such case to switch the $\mathrm{Q}$ to dampen the ringing [19].

In most of the modern circuits, a single coil is used for both excitation of the nuclei and detection of signals. This poses some stringent requirement of the probes and additional circuitry to isolate the excitation and detection circuits.In some of the designs $[20,21]$ the transmitter and receiver coils are different, which simplifies the electronics and also makes it easier to optimize the independent requirements of both transmitter and receiver. However, mechanically, the cross coil arrangement is more complicated than the single coil system because a larger volume is required in the crossed coil arrangement. Moreover isolating the transformer coupling between the excitation coil and the receiving coil could be quite a challenge.

Several designs have been proposed for NQR coil. For lab designed spectrometers, a simple solenoid is often employed, where the sample under study is placed inside the coil that enables the effective utilization of the magnetic fields. For field applications requiring the detection of chemical compounds, a flat pattern coil is often used. There are also some coil designs that provide more robust interference rejection [22]. In order to make the receiver configuration simpler, it is observed that the parallel tank circuit is more desirable for an NQR probe.

The probe circuit has a coil and two variable capacitors. With the adjustment of capacitors the probe circuit is configured so that the tuning is achieved over the desired frequency range with an input impedance of the probe circuit at 50.The forward power generated by power amplifier is transferred to the probe, by matching the impedance of probe to $50 \mathrm{ohm}$. Also the signal generated by probe will be efficiently transferred to detection circuit as the probe impedance is matched to $50 \mathrm{ohm}$ which is the input impedance of pre-amplifier.The two probe circuits, series tuned circuit and parallel tuned circuit are discussed here. The parallel tuned LC circuit impedance matched to $50 \mathrm{ohms}$ with a series capacitor is shown in Figure 3. The capacitors are varied in order to tune the circuit to the resonance frequency and match the circuit to 50 ohms for maximum power transfer from the transmitter. For the circuit shown in Figure 3. The impedance between $\mathrm{A}$ and $\mathrm{C}$ equals:

$$
\begin{aligned}
& Z_{A C}=Z_{B C}+\frac{1}{j \omega C_{M}}=\frac{(R+j \omega L) / j \omega C_{T}}{R+j \omega L+1 / j \omega C_{T}}+\frac{1}{j \omega C_{M}} \\
& =\frac{R}{\left(1-\omega^{2} L_{T}\right)^{2}+\left(\omega C_{T} R\right)^{2}}+j \omega \frac{L\left(1-\omega^{2} L C_{T}\right)-C_{T} R^{2}}{\left(1-\omega^{2} L C_{T}\right)^{2}+\left(\omega C_{T} R\right)^{2}}+\frac{1}{j \omega C_{M}}
\end{aligned}
$$




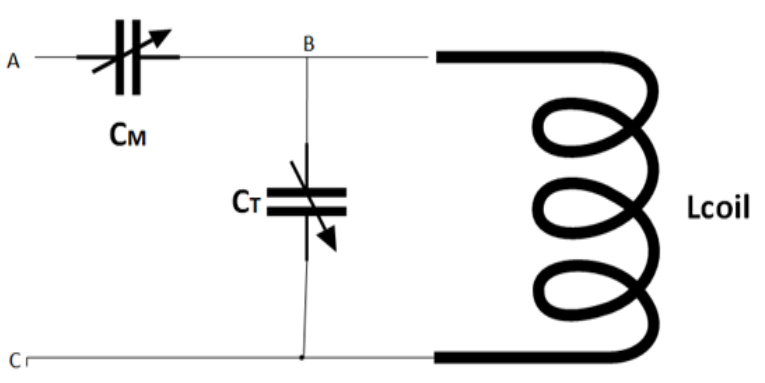

Figure 3. Probe with parallel tuned circuit

$\mathrm{R}$ is the resistance of coil at resonance frequency. Since the terms $C_{T} R^{2}$ and $\left(\omega C_{T} R\right)^{2}$ are very small and negligible, as shown in (2) can be simplified to:

$$
Z_{A C}=\frac{R}{\left(1-\omega^{2} L C_{T}\right)^{2}}+j \omega \frac{L}{\left(1-\omega^{2} L C_{T}\right)}+\frac{1}{j \omega C_{M}}
$$

In order to satisfy the matching condition the real part of (3) must be set to characteristic impedance of the connecting coaxial cable which is $50 \Omega$ by adjusting $C_{T}$ at fixed frequency:

$$
\frac{R}{\left(1-\omega^{2} L C_{T}\right)^{2}}=50 \Omega
$$

Thus, $\quad C_{T}=\frac{1-\sqrt{R / 50}}{\omega^{2} L}$

In order to obtain resonance at same frequency imaginary part of (3) must be zero. Thus equating imaginary part of (3) to zero, we get:

$$
C_{M}=\frac{\sqrt{R / 50}}{\omega^{2} L}
$$

The probe circuit with series tuned LC circuit impedance matched to 50 ohms with a parallel capacitor is shown in Figure 4.

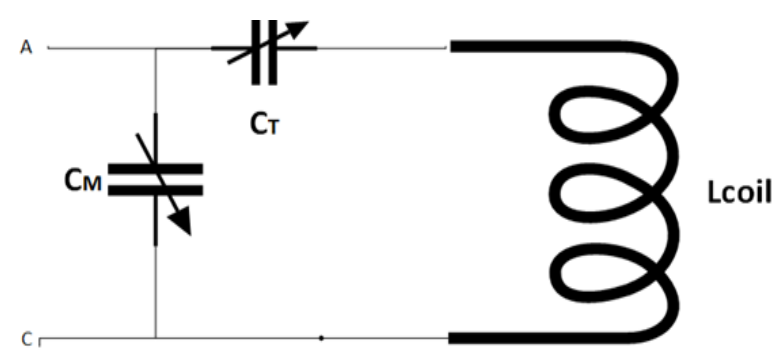

Figure 4. Probe with series tuned circuit

For the circuit shown in Figure 4 the impedance between $\mathrm{A}$ and $\mathrm{C}$ equals:

$$
\begin{aligned}
& Z_{A C}=\frac{\left(R+j \omega L+\frac{1}{j \omega C_{T}}\right) * \frac{1}{j \omega C_{M}}}{\left(R+j \omega L+\frac{1}{j \omega C_{T}}\right)+\frac{1}{j \omega C_{M}}} \\
& Z_{A C}=\frac{R C_{T}^{2}}{\omega^{2} C_{T}^{2} C_{M}{ }^{2} R^{2}+\left(\omega^{2} L C_{T} C_{M}-C_{T}-C_{M}\right)^{2}}-j \frac{\omega^{2} C_{T}{ }^{2} C_{M} R^{2}+\left(\omega^{2} L C_{T}-1\right)\left(\omega^{2} L C_{T} C_{M}-C_{T}-C_{M}\right)}{\omega^{2} C_{T}{ }^{2} C_{M}{ }^{2} R^{2}+\left(\omega^{2} L C_{T} C_{M}-C_{T}-C_{M}\right)^{2}}
\end{aligned}
$$


$\mathrm{R}$ is the resistance of coil at resonance frequency. Since the terms $\left(\omega^{2} L C_{T} C_{M}-C_{T}-C_{M}\right)^{2}$ very small and negligible, as shown in (7) can be simplified to:

$$
Z_{A C}=\frac{R}{\omega^{2} C_{M}^{2} R^{2}}-j \frac{\left(\omega^{2} L C_{T}-1\right)\left(\omega^{2} L C_{T} C_{M}-C_{T}-C_{M}\right)}{\omega^{2} C_{T}^{2} C_{M}^{2} R^{2}}
$$

In order to satisfy the matching condition the real part of (8) must be set to characteristic impedance of the connecting coaxial cable which is $50 \Omega$ by adjusting $C_{T}$ at fixed frequency:

$$
\frac{R}{\omega^{2} C_{M}^{2} R^{2}}=50 \Omega
$$

Thus, $\quad C_{M}=\frac{1}{\omega \sqrt{50 R}}$

In order to obtain resonance at same frequency imaginary part of (8) must be zero. Thus equating imaginary part of (8) to zero, we get:

$$
C_{T}=\frac{1}{\omega^{2} L}
$$

\section{DESIGN OF COIL}

The RF coil combines with matching circuit to form a resonant circuit, and works under the resonant state) [23]. Ideal coil should possess uniform RF field, good fill factor and high quality factor (Q). The amplitude of RF voltage which appears at the terminal of the tank circuit at resonance frequency is proportional to quality factor of the coil thus it is advisable to use coils with high Q factor [24]. The quality factor is known as $Q$ value and it represents the ratio of inductive reactance and equivalent loss resistance.

$$
Q=\frac{2 \pi f L}{R}
$$

Where $f$ is the frequency, $L$ is the inductance of the coil, and $\mathrm{R}$ is the total loss resistance. The $Q$ value is higher, the loss of the circuit is smaller, the signal amplification is stronger, the frequency selectivity is better, and frequency stability of resonant circuits is higher. However the bandwidth becomes very narrow at high $Q$ resulting in excessive loss of useful signal spectral components and energy. Thus the key design of coil is the selection of $Q$ and the range of the variable capacitors. Several coils were wound and tested. The one that was finally used for this work was with length to diameter ratio of two [25], is made out of 17 AGW copper wire and has 32 turns of $20 \mathrm{~mm}$ diameter of length $40 \mathrm{~mm}$.

Smith Software is used to find the range of inductance of coil with available capacitors $C_{T}$ and $C_{M}$ of $(5 \mathrm{pF}-125 \mathrm{pF})$. The capacitors used are high voltage $(5 \mathrm{kV})$ vacuum variable capacitors. According to inductance, the coil diameter and the length of turns, the turns of the coil winding were calculated by induction calculation software. The coil was designed and made mechanical stable by potting its turns in resin based adhesive (Araldite ${ }^{\circledR}$ ). The inductance of the coil is $8 \mu \mathrm{H}$.

\section{DESIGN OF PROBE}

For observing ${ }^{14} \mathrm{~N}$ NQR signal from $\mathrm{NaNO}_{2}(\mathrm{NQR}$ Frequency $=4.64 \mathrm{MHz})$ and $\mathrm{L}=8 \mu \mathrm{H}$. The value of capacitors for parallel tuned circuit probe as per (5) and (6) are $\mathrm{C}_{\mathrm{T}}=126.4 \mathrm{pF}, \mathrm{C}_{\mathrm{M}}=20.8 \mathrm{pF}$, Also simulated using Smith software as shown in Figure 5 the values of $\mathrm{C}_{\mathrm{T}}$ and $\mathrm{C}_{\mathrm{M}}$ are $126.5 \mathrm{pF}$ and $20.7 \mathrm{pF}$ respectively. The value of capacitors for series tuned circuit probe as per (10) and (11) are $\mathrm{C}_{\mathrm{T}}=147 \mathrm{pF}, \mathrm{C}_{\mathrm{M}}=4.85 \mathrm{nF}$. Also simulated using Smith software as shown in Figure 6 the values of $C_{T}$ and $C_{M}$ are $151.8 \mathrm{pF}$ and $4.8 \mathrm{nF}$. The parallel tuned LC circuit probe looks fine for the work as values of capacitances are matching to values of available capacitors. The values of capacitors in series probe are not as per the ratings of available capacitances and also the size of variable capacitor of range in $\mathrm{nF}$ is too large. For a current pulsed NQR spectrometer parallel tuned circuit probe with coil of $8 \mu \mathrm{H}$ was developed and photo is shown in Figure 7 . The probe circuit is shielded inside an aluminum box and is well grounded to reduce the noise. The coil is made mechanically stable by binding its turns using a resin based adhesive (Araldite ${ }^{\circledR}$ ). The forward power is maximised and reflected power is minimised by tuning and matching of the probe. The forward and reflected power is observed by directional coupler built in the transmitter. Figure 8 shows the plot of impedance of probe versus frequency. 


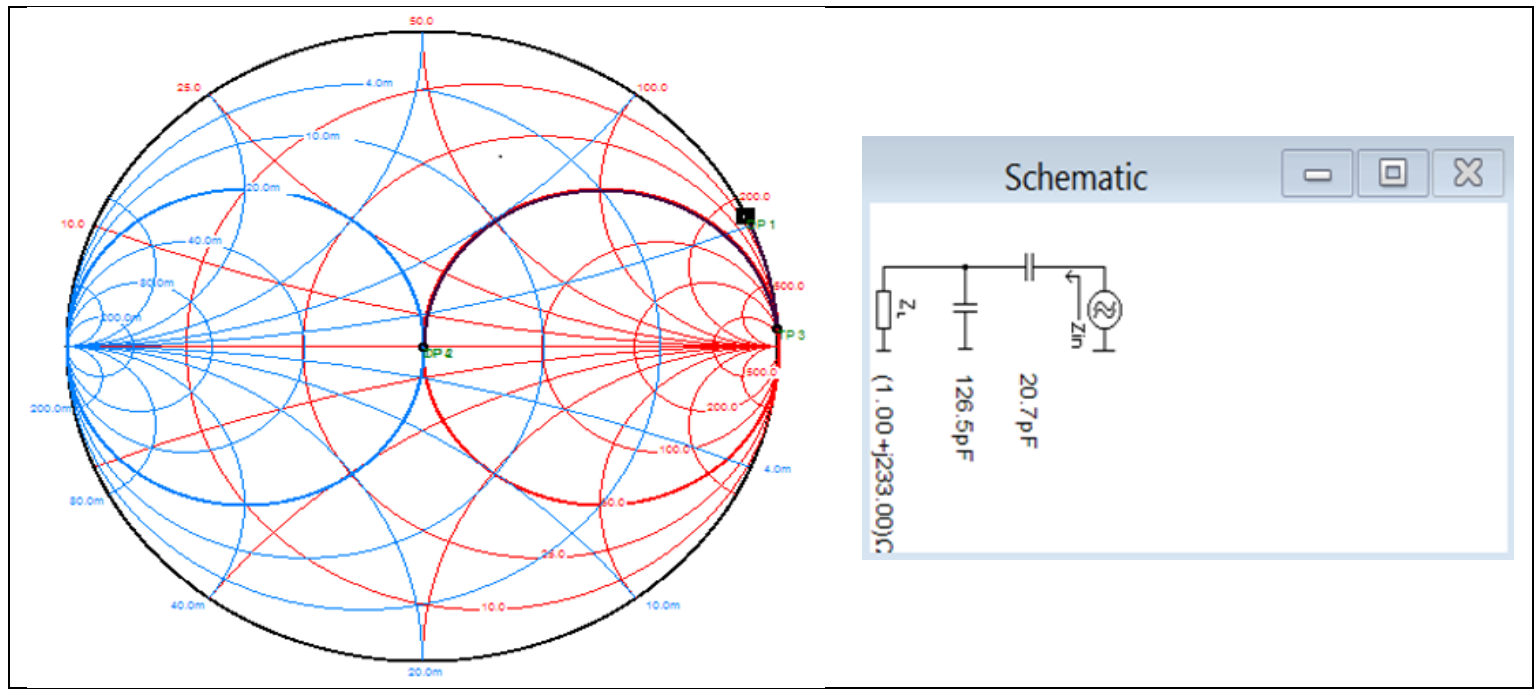

Figure 5. Smith chart and schematic of probe with parallel tuned circuit obtained from smith software

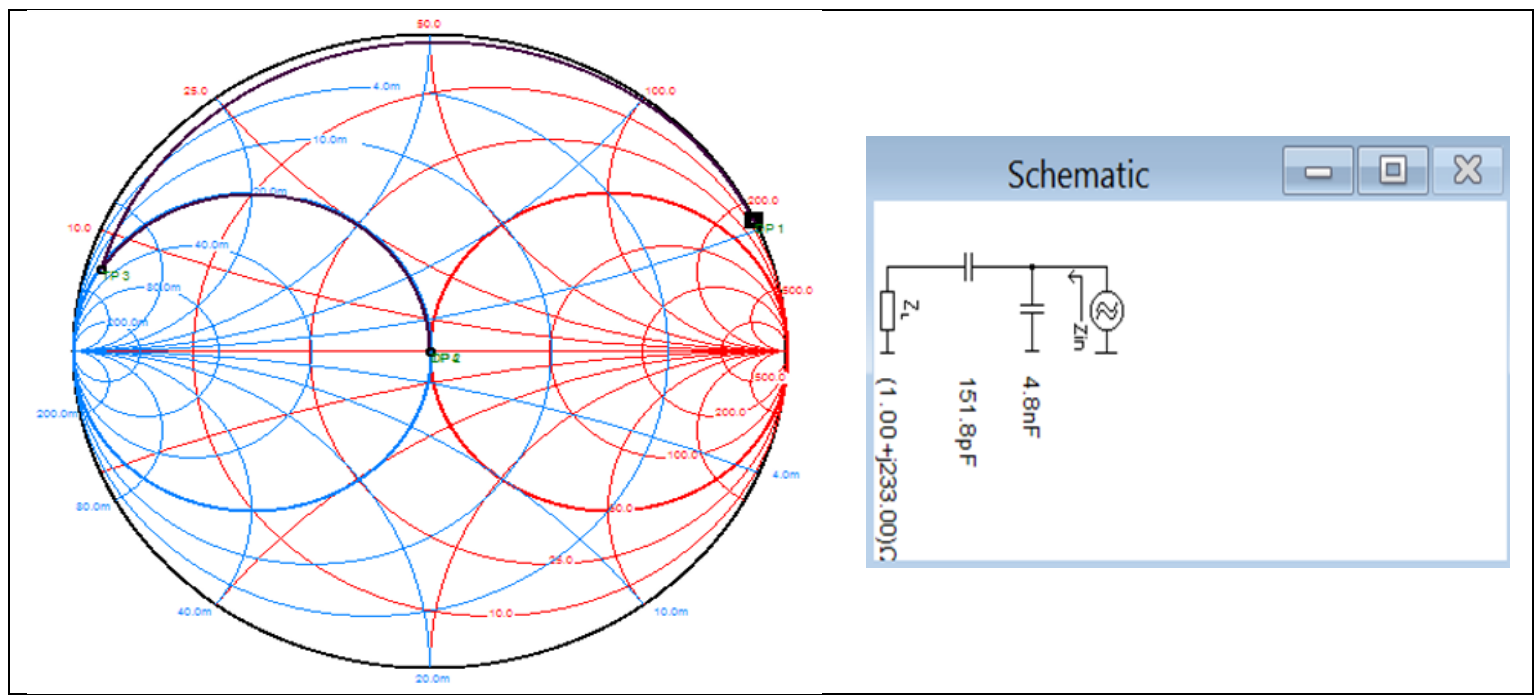

Figure 6. Smith chart and schematic of probe with parallel tuned circuit obtained from smith software

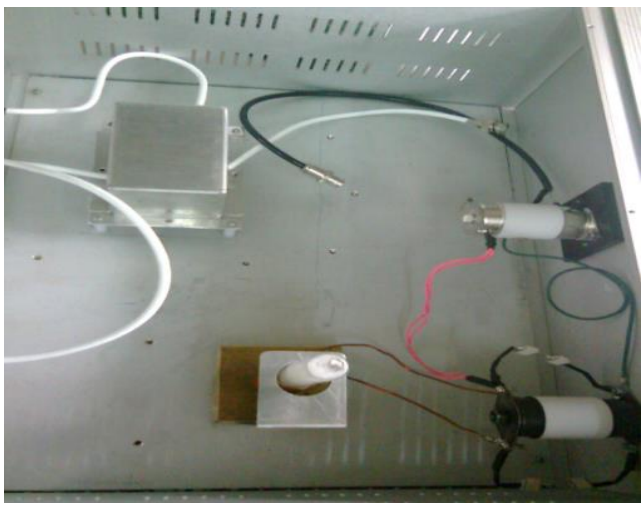

Figure 7. Photograph of parallel tuned circuit probe

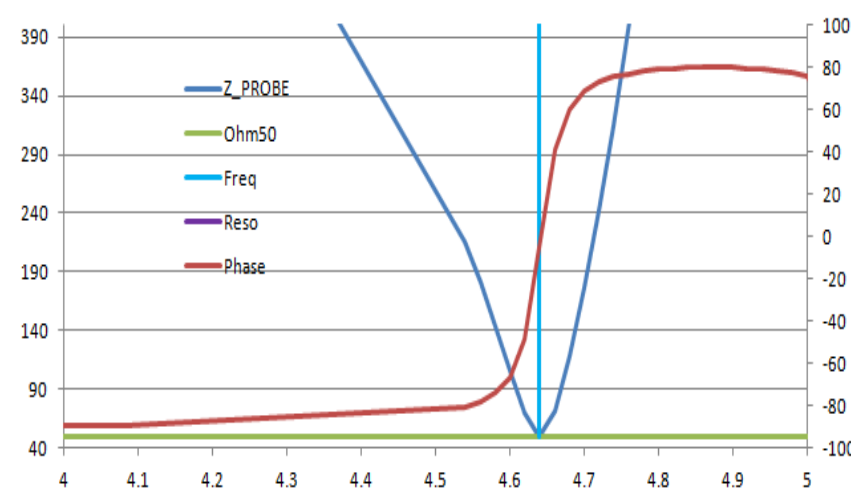

Figure 8. impedance of probe vs frequency. horizontal axis is frequency in $\mathrm{MHz}$, vertical axis in left is $\mathrm{Z} \_$probe in ohms, vertical axis in right is phase in degrees 


\section{RESULTS}

\section{1. ${ }^{14} \mathrm{~N} N Q R$ signal}

FPGA based NQR spectrometer using parallel tuned probe circuit was designed and developed. It was used to observe ${ }^{14} \mathrm{~N}$ NQR signal from $\mathrm{NaNO}_{2}$. Figure 9 shows the ${ }^{14} \mathrm{~N} \mathrm{NQR}$ signal. The observation frequency is $4.642 \mathrm{MHz}$ where as the signal is off resonant by about $2500 \mathrm{~Hz}$ resulting in oscillatory NQR signal. RF excitation pulse length of $20 \mu \mathrm{s}$ and power of $120 \mathrm{~W}$ was used for excitation. The pulse length is selected such that it is a $\pi / 2$ pulse, to get maximum amplitude of NQR signal.

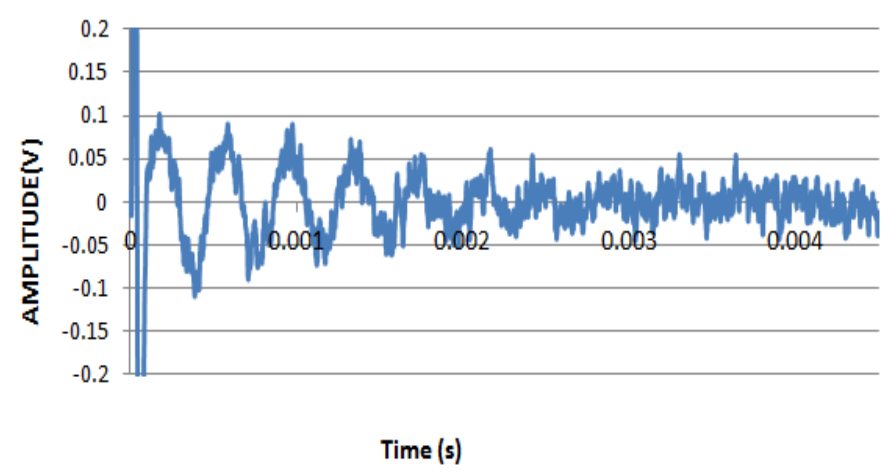

Figure 9. ${ }^{14} \mathrm{~N} \mathrm{NQR}$ signal from $\mathrm{NaNO}_{2}$

\section{CONCLUSION}

An FPGA based pulsed NQR spectrometer is explained in this paper. The entire digital task required for spectrometer such as Pulse Programmer, DDS, Digital Receiver, a low pass FIR filter and data acquisition is developed in FPGA. Also, the analog peripheral components such as pre-amplifier, cross diodes, quarter wave transformer and parallel tuned probe were designed and developed. Series and parallel tank circuits were simulated. The simulation shows that range of variable capacitors required for parallel tuned circuit are of lower range (so easily available) as compared to that required for series tuned circuits.

\section{REFERENCES}

[1] Garrowway A. N, Buess M. L, Miller J. B, Suits B. H, Hibbs A. D, Barrall G. A, Matthews R. and Burnett L. J, "Remote sensing by nuclear quadrupole resonance," in IEEE Transactions on Geoscience and Remote Sensing, vol. 39, no. 6, pp. 1108-1118, June 2001.

[2] Shengyun Z., Xing L., Zhongcheng L., Guobao W., and Weiguo S., "Application of Nuclear Quadrupole Resonance to Detection of Explosives and Research Activities at CIAE," International Atomic Energy Agency (IAEA), vol. 43, no. 18, 2010.

[3] E. Gudmundson, A. Jakobsson and P. Stoica "NQR based Explosive detection-An Overview," $9^{\text {th }}$ International symposium on signal Circuits and systems (ISSCS), Iasi, Romania, July 2009.

[4] P. Hemnani, G. Joshi, A. K. Rajarajan and S. V. G. Ravindranath, "14N NQR spectrometer for explosive detection: A review," 2016 International Conference on Automatic Control and Dynamic Optimization Techniques (ICACDOT), Pune, pp. 1120-1125, 2016, Doi: 10.1109/ICACDOT.2016.7877761.

[5] W. L. Rollwitz, J. D. King, and G. A. Matzkanin, "Fundamentals of nuclear magnetic resonance for the detection and identification of explosives," Proceedings of New Concepts Symposium and Workshop on detection and Identification of Explosives, 1978.

[6] Flexman J. H., Rudakov T. N., Hayes P. A., Shanks N., Mikhaltsevitch V. T., Chisholm W. P., "The Detection of Explosives in Airport Luggage Using the Direct Nuclear Quadrupole Resonance Method" In: Schubert H., Kuznetsov A., "Detection of Bulk Explosives Advanced Techniques against Terrorism," NATO Science Series (Series II: Mathematics, Physics and Chemistry), vol 138, Springer, Dordrecht, 2004.

[7] Rabi, I. I., Zacharias, J. R., Millman, S., Kusch, P., "A New Method of Measuring Nuclear Magnetic Moment," Physical Review Journals Archive, vol. 53, no. 4, pp. 318-318, February 1938.

[8] Glase S. J., "NMR Quantum Computing," Angewandte Chemie International Edition, vol. 40, no. 1, pp. 147-149, 2001.

[9] Hemnani P., Rajarajan A. K., Joshi G., Ravindranath S. V. G., "FPGA based Pulsed NQR spectrometer," AIP conference Proceedings, vol. 1591, no. 1, pp. 661-663, 2014.

[10] Kazuyuki J. S., "Humanitarian de-mining: Detection algorithms for NQR signals," M.S. Thesis, University of Twente, Netherland, January 2004. 
[11] Kazuyuki T., "OPENCORE NMR: Open-source core modules for implementing an integrated FPGA-based NMR spectrometer," Journal of Magnetic Resonance, vol. 192, no. 2, pp. 218-229, 2008.

[12] Hemnani P., Rajarajan A. K., Joshi G., Ravindranath S. V. G., "The Building of pulsed NQR/NMR Spectrometer," International Journal of Electrical and Computer Engineering (IJECE), vol. 8, no. 3, pp. 1442-1450, June 2018.

[13] Hemnani P., Rajarajan A. K., Joshi G., Ravindranath S. V. G., "Development of Low frequency pulsed NMR/NQR spectrometer," Global Journal of Research in Engineering, vol. 16, no. 2, 2016.

[14] Hemnani P., Rajarajan A. K., Joshi G., Ravindranath S. V. G., "FPGA based RF pulse generator for NQR/NMR spectrometer," Procedia Computer Science, vol. 93, pp. 161-168, 2016.

[15] Eva M., Colm S., “All about Direct digital synthesis,” Analog Dialogue, vol. 38, August 2004.

[16] João G., Jorge R. F., Manuel M. S., “A Reconfigurable quadrature oscillator based on a DDS system,” DCIS 2006, [Online]. Available. http://www.inesc-id.pt/ficheiros/publicacoes/3627.pdf

[17] Fukushima, R., "Experimental Pulse NMR: Nuts and Bolts approach," Westview Press, 1993.

[18] Gurpadam S., Neelam R. P., "FPGA Implementation of Higher Order FIR Filter," International Journal of Electrical and ComputerEngineering (IJECE), vol. 7, no. 4, pp.1874-1881, August 2017.

[19] Mark S. C., "FET Q switch for pulsed NMR," Review of Scientific Instruments, vol. 48, issue 3, pp. 359-361, March 1977, Doi: 10.1063/1.1135027

[20] Grechishkin V. S., "NQR device for detecting plastic explosives, mines, and drugs," Applied Physics A, vol. 55, no. 6, pp. 505-507, 1992.

[21] Rudakov T. N., Belyakov A. V. and Mikhaltsevich V. T., "A low-frequency instrument for remote nuclear quadrupole resonance experiments," Measurement Science and Technology, vol. 8, no. 4, pp. 444-448, 1997.

[22] Suits B. H., "The noise immunity of gradiometer coils for $14 \mathrm{~N}$ NQR land mine detection: practical limitations," Applied Magnetic Resonance, vol. 25, no. 3, pp. 371-382, September 2004.

[23] Hao, F., Xu G., Huang X., "Study of RF Coil in NQR Explosive Detection System," Procedia Engineering, Vol. 43, pp. 302-306, 2012.

[24] Alan G., Tomaž A., "Aplicability of TNT "super-QQ detection" to multipulse sequences," Journal of Magnetic Resonance, vol. 201, no. 2, pp. 131-136, September 2009.

[25] Minard K. R., Wind. R. A., "Solenoidal Micro coil Design-Part1: Optimizing RF Homogeneity and Coil Dimensions," Concepts in Magnetic Resonance, vol. 13, no. 2, January 2001. 